\title{
Zinc Coating Obtained in the Result of the Mechanochemical Effects in Vibrodynamic the Setups of Various Types
}

\author{
Vladimir Ivanov ${ }^{1}$, Sergey Popov ${ }^{1}$, Nikolay Dontsov ${ }^{1}$, Dieudonné Essola ${ }^{2}$ \\ ${ }^{1}$ Don State Technical University, Gagarin square 1, 344000, Russia \\ ${ }^{2}$ University of Douala. BP 2701Douala, Littoral Province, Cameroon
}

\begin{abstract}
The results of investigations of zinc coatings obtained on the surface of metals in the process of mechanochemical synthesis realized in conditions of vibro-wave technological systems (VTS) are presented. The features of the morphology of the coating structure are revealed, which activates the role of indents in the formation of free-moving vibrations under the influence of low-frequency oscillations, and its influence on increasing the operational properties of the surface layer of the parts. The advantages of this method of applying zinc coatings in comparison with traditional methods are shown.
\end{abstract}

\section{Introduction}

The formation of a zinc coating on the surface of a metal under conditions of vibro-wave technological systems is a complex process that is the result of a joint action of a mechanical component and a chemical reaction. When coating mechanically and chemically, one of the main indicators of surface quality is the absence of a hydrogenated layer. The purpose of the research is to establish the technological features of the formation of the mechanochemical zinc coating formed by the vibration-effect of free-moving indentors.

\section{Materials and methods}

The process of applying zinc coating mechanically and chemically in rotating drums has a strong reputation in the world. When coating is applied in such a way, one of the main quality indicators is the absence of a hydrogen charged layer. However, the use of this equipment limits the scope of application because of its specialization. The wide technological possibilities of vibration processing are the basis for the creation on the surface of parts of coatings of various types and purposes. However, each kind of coating has its own physical-chemical properties, and is formed according to the laws inherent only to it. Therefore, the disclosure of the physical nature of the process requires additional studies of surface morphology.

The formation of a zinc coating on the metal surface is a complex process resulting from the combined effect of the mechanical component and the chemical reaction. 
Taking into account the features of the vibration processes, a technical process for the formation of the zinc coating was developed.

In the process of vibratory action, the metal surface is subjected to numerous differently directed impacts, elastoplastic deformation and, as a result, surface activation; at the same time, there are such phenomena as adsorption and diffusion.

At the first moment of coating formation, the zinc ion from the solution passing through a double layer is freed from the hydrated shell and is adsorbed on the active surfaces of the protrusions.

Here the zinc ion $\left(\mathrm{Zn}{ }^{++}\right)$is discharged (takes electrons) and $(\mathrm{Zn})$ is deposited on the metal surface due to oxidation-reduction processes in the form of a zinc coating. The formation of the first crystals of the precipitated metal leads to the formation of micro / nano "coating-metal" elements. As a result, a field of trace elements is superimposed on the potential difference between Fe- Zn. An increase in the internal energy of the surface layers of the metal, as a result of plastic deformation, leads to an increase in the metal surface absorption activity, the activity of ions and molecules, an increase in the crystallization centers and the density of microcurrents, while the micro / nano structures of the zinc coating are introduced into the coating. The resulting EMF exerts a significant influence on the further course coating formation. $[1,-5]$.

The formation of crystalline coating structures occurs not only on the protrusions, but also on the facets and in the indentations. As a result, the metal is coated more evenly, the crystals are closed, forming a continuous coating. The groups of zinc coating crystals are clearly visible in Fig.1

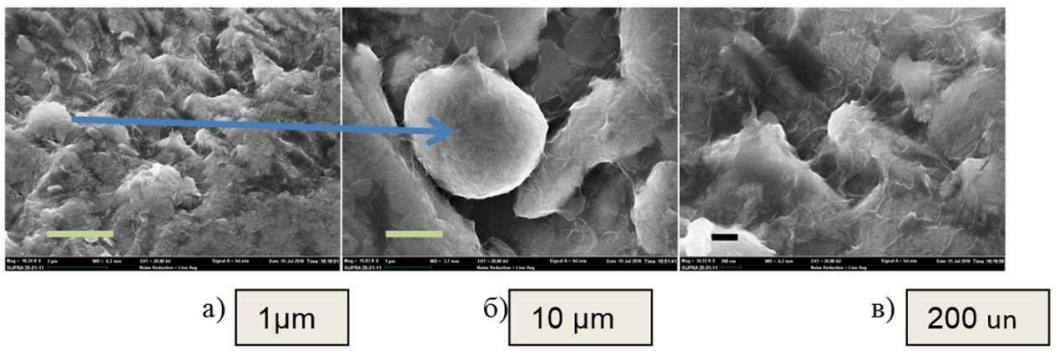

Fig. 1 Vibration mechanical-chemical zinc coating

The coating layer formed in the initial stage is the basis for its subsequent growth, it is very important to ensure maximum surface activity. The formation of a large number of small crystals provides a more complete overlapping of the base metal. As a result, less porosity, increased corrosion resistance and greater adhesion strength to the base metal are achieved. [5-9].

Interaction at the phase interface leads to the appearance of a difference in the compositions, surface and inner layers of this phase and, consequently, to the process of equalization of their composition in the Fe- Zn system. The latter is accelerated by convection processes with VMCZC.

The desire to equalize the concentrations entails a diffusion process, which is determined by the thermal motion of atoms (molecules), temperature gradients, electric fields, etc.

Investigation of the electron image of the vibrational mechanical-chemical coating section (Fig. 2) and the transition zone spectral analysis revealed that there is no sharp boundary (metal-coating), inclusions in the depth of the base metal of Fe- $\mathrm{Zn}$ up to 4 microns were found. 


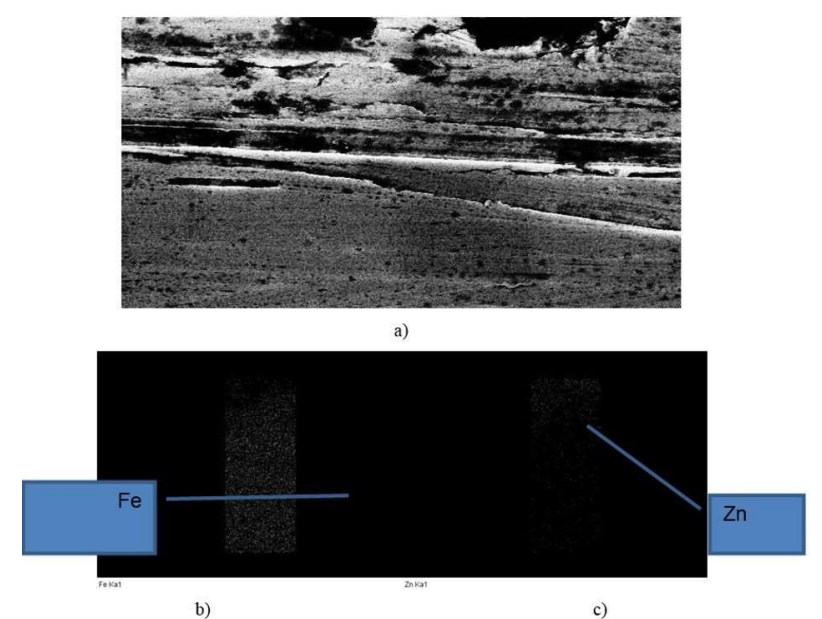

Fig.2. Thin section VMCZC: a)general view; b) spectral imageof the main metal Fe;c)spectral image of the coating $\mathrm{Zn}$

The method of obtaining zinc coatings is the main factor determining their structure and properties. The microstructure of the zinc coating, obtained by galvanic treatment and under the conditions of HHC, is shown in Fig 3. The sharp boundary between the steel base and the zinc layer is visible (Fig. 3a, b), i.e., for this galvanizing process, the base metal and the coating together form a compound at the molecular level, since the process proceeds at a low temperature, not sufficient for intensive diffusion. The electrodeposited coating presented for comparison is characterized by porosity, a sharp metal-substrate boundary, the presence of a hydrogenated layer, which worsens its operation compared to VMCZC [10-14].

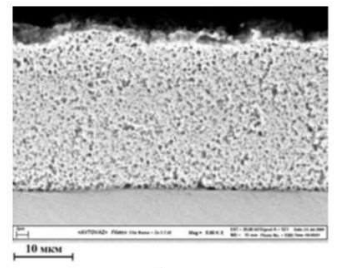

a)

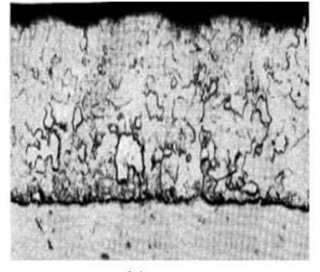

b)

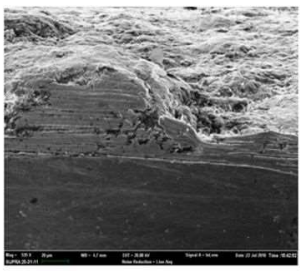

Fig.3. Galvanic zinc coating, obtained: a- in the stationary application mode; b-section of VMCZC; c galvanic coating X-300; in-VMCZC.

The structure of the vibrational mechanical-chemical coating, as seen from the spectrogram and the section, has a nonporous structure and a sharp boundary between the metal and the coating, which explains its increased corrosion properties and is a distinguishing feature of VMCZC and an undoubted advantage over galvanic coatings. [1517].

Zinc plating in the process of vibrational mechanical-chemical treatment is used for structural parts of carbon and alloyed steels. The equipment can be serially produced vibrating machines with rectangular and circular working chambers (Fig. 4a, b). 


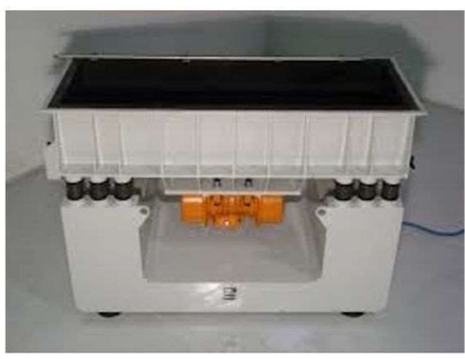

a)

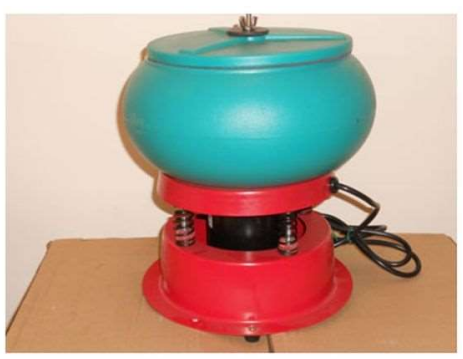

b)

Fig.4. Vibrating machines: a - rectangular working chamber; $b$ - annular working chamber. Glass, porcelain balls with a diameter of 2-8 mm, zinc powder (PC-2) (Fig. 5) and chemical activators are used as the working medium.

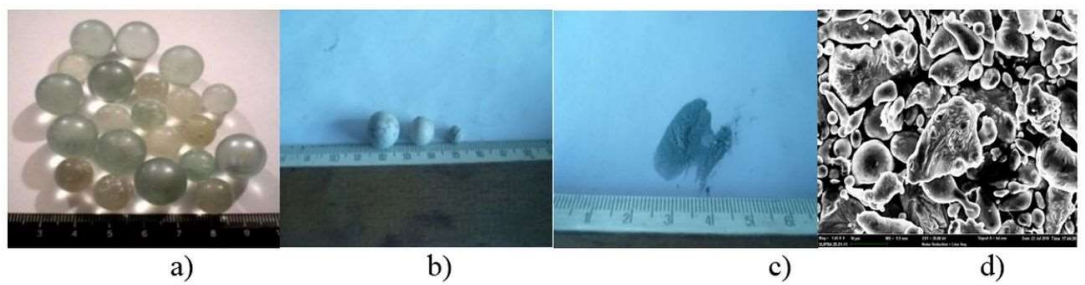

Fig.5. Examples of working media: a, - glass; b - porcelain; c- zink powder (PC-2); d-zinc powder scale of 10 microns.

The coating process is carried out in the process fluid. The thickness of the zinc coating is up to $20 \mu \mathrm{m}$ and in comparison with the traditional galvanic zinc coating it has got:

- low power consumption of technology;

- ecological purity;

- lack of surface hydrogenation;

-good physical-mechanical and operational properties of the surface: low roughness;

- high corrosion resistance of the coating.

The combined process of vibrational mechanical-chemical galvanizing can be considered as an aggregate of independent influence of mechanical, chemical and mechanical-chemical factors.

The process of VMCZC is affected by the processing modes; frequency and amplitude of oscillations; composition and abrasive properties of the working medium; volume ratio of the working environment and parts; constructive relationships of the machined parts and the working chamber. [6].

The thickness of the coating in this case is determined by the difference in the rates of formation of the coating and the concomitant removal of the metal. In turn, the formation of the coating is also determined by mechanical factors. During the coating phase, the cleaned and washed parts were loaded into a hermetically sealed working chamber with porcelain balls $5-10 \mathrm{~mm}$ in diameter, a solution containing zinc chloride in an amount of 100 grams per liter of water, and zinc powder (50 grams per liter of solution). The temperature of the working solution can range from $180-350^{\circ} \mathrm{C}$, at low temperatures of $00-150$ the coating time is increased by $15 \%$. Operating modes of the equipment are set within the following limits: amplitude of oscillation $3 \mathrm{~mm}$, oscillation frequency $25 \mathrm{~Hz}$, time 45-60 min. [7, 8].

After coating, the parts were removed from the chamber, washed with cold running water, and dried. Fig. 5 presents samples of parts used in the automotive industry, this method is applicable both for large enterprises and for small repair shops. 

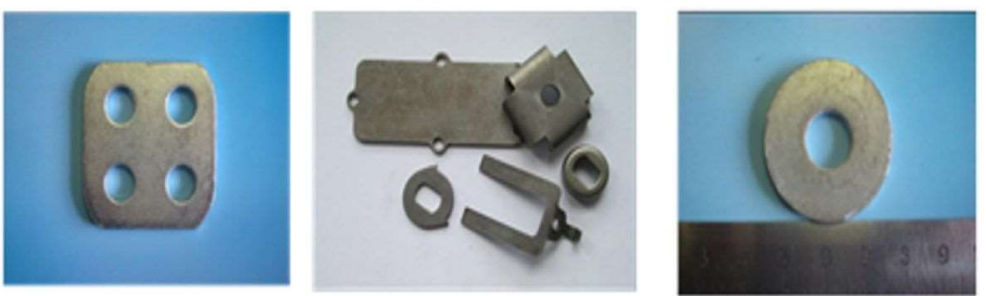

Fig.5. Samples of parts with vibrating mechanical-chemical coating.

In order to increase corrosion resistance, zinc coatings were subjected to special chemical treatment in a chromate solution of composition, g / 1: nitric acid (HNO3) -2-5; anhydrite chromic (CrO3) - 25-55; sodium sulphate (Na2SO4) - 15-20.

The treatment was carried out at a temperature of $15-30^{\circ} \mathrm{C}$ for $0.1-0.3$ minutes. Then the parts were washed in cold running water. The parts were dried for 6 minutes at a temperature of $50^{\circ} \mathrm{C}$. $[9,10]$.

Vibrational mechanochemical coatings are almost non-porous, therefore, chromatic treatment can be avoided.

\section{Conclusions}

1. Zinc coating obtained in the conditions of vibration technology systems has a number of advantages, such as the absence of a hydrogenated layer, low porosity, high corrosion resistance.

2. Coating technology does not require special equipment, facilities, highly qualified specialists, special equipment for cleaning and recycling of waste.

3. Applications of this method of zinc coatings are advisable for parts without deep holes.

\section{Reference}

1. A.P. Babichev and I.A. Babichev, Fundamentals of Vibration Techniques, (DSTU Publishing House, Rostov-on-Don, 2008)

2. A. Brooks, Metal Finishing. 81:8, 53 (2008)

3. Rouabhi Youcef, Lounis Azzeddine, A. P. Babichev, J. New Technol. Mater., 6:2 (2016)

4. Jess Russell, Mechanical plaiting (2012)

5. L. K. Gillespie, The formation and properties of machining burrs, M.S. thesis, (Utah State University, Logan, Utah, USA, 1973)

6. V.V. Ivanov, S.I. Popov, A.V. Kirichek, X I International Conference on Mechanical Engineering, Automation and Control Systems (MEACS 2017) 327 (2018)

7. Gillespie La Roux, K Mass, Finishing Handbook, Society of Manufacturing Engineers Dearborn, (Michigan USA SME, 2006)

8. V.V. Ivanov, N.S. Dontsov, A.V. Kirichek, Key Engineering Materials, 736, 105 (2017)

9. V. V. Ivanov, S.I. Popov, A.V. Kirichek, Key Engineering Materials, 736, 18 (2017)

10. V.V. Ivanov, N.S. Dontsov, A.V. Kirichek, XI International Conference on Mechanical Engineering, Automation and Control Systems (MEACS 2017) 327 (2018)

11. V. A. Lebedev, V.V. Ivanov and V. P. Fedorov, Materials Science and Engineering 124 (2016) 
12. A. Babichev, V. Ivanov, Pogoelov, The research of technological characteristic of the vibrowave mechanical and chemical oxide coating formation (2017)

13. A. A. Ivakhnenko, M.L. Storublev, A.G. Ivakhnenko, Procedia Engineering, 150 (2016)

14. Valeriy Blumenstein, Maksim Mahalov, Oleg Ostanin, E3S Web of Conferences 41, $03012(2018)$

15. V.V. Ivanov., V. P. Smolentsev, A. I. Portnykh, MEACS 2017 IOP Publishing. IOP Conf. Series: Materials Science and Engineering 327 (2018)

16. V. Ivanov, N. Pogorelov, M. Selemenev, A. Sakunov, MATEC Web of Conferences (2018)

17. Dieudonné Essola Jean Chills Amba2, Claude Valery Ngayihi Abbe,Joseph Nkongho Anyi, V.V. Ivanov, Journal of Mechanical and Materials Engineering volume 14, 8 (2019) 